\title{
SMART LEGAL CONTRACTS: EINSATZMÖGLICHKEITEN IM RECHT UND RECHTLICHE RAHMENBEDINGUNGEN ${ }^{1}$
}

\author{
SMART LEGAL CONTRACTS: POSSIBILIDADES DE APLICAÇÃO E ENQUADRAMENTO LEGAL
}

\author{
SMART LEGAL CONTRACTS: POSSIBILITIES OF APPLICATION AND LEGAL FRAMEWORK
}

Gustavo Marchi de Souza Mello*

\begin{abstract}
Zusammenfassung:
Das Ziel dieser Arbeit besteht darin, die neue Kategorie des smart contracts, d.h. automatische Softwareprogramme, die auf Blockchain-Plattform aufgebaut sind, aus rechtlicher Sicht zu untersuchen. Genauer gesagt wird untersucht, wie sie sich potenziell auf das Vertragsrechtsuniversum auswirken können, indem sie als Antwort des Rechts auf die Herausforderungen einer Gesellschaft in ständiger Entwicklung dienen. Insgesamt widmet sich die vorliegende Arbeit der Frage, ob diese Verträge tatsächlich ein innovatives Rechtsinstrument darstellen oder nicht eine aufkeimende Frage, die noch einer Antwort bedarf. Die eigentlich rechtliche Seite der technisch sogenannten smart contracts, die in diesem Zusammenhang von Synergien zwischen Recht und Technik als smart legal contracts zu bezeichnet sind, soll geklärt werden. Auf diese Weise werden sowohl (i) seine rechtlichen Rahmenbedingungen, als auch (ii) seine Einsatzmöglichkeiten im deutschen Recht, insbesondere was das Vertragsrecht betrifft, analysiert. Diese Untersuchung ist vor allem aufgrund des innovativen Potentials dieser obengenannten „Verträge“ in der Dynamik des Segments gerechtfertigt - unter den möglichen Innovationen kann man die Vereinfachung und Automatisierung von Vorgängen kraft des in den smart contracts innewohnenden Selbstausführungsmechanismus erwähnen.
\end{abstract}

Stichwörter: Smart Legal Contracts. Verträge. Automatische Programme. Blockchain. Selbstausführbarkeit.

\begin{abstract}
Resumo:
O presente trabalho tem por escopo estudar, sob o prisma jurídico, a recente figura dos smart contracts, programas automáticos de software construídos sobre plataformas blockchain. Mais especificamente, estuda-se como eles poderão impactar o universo jurídico contratual, servindo como a resposta do Direito aos desafios que a sociedade, em constante evolução, lhe propõe. Em linhas gerais, investigar-se-á se os referidos contratos efetivamente são uma ferramenta jurídica inovadora ou não - questão que, dado o seu caráter incipiente, ainda não está resolvida. Espera-se esclarecer a faceta
\end{abstract}

1 Das Thema wurde im Winter 2018 im Rahmen des Seminars „Synergien zwischen Rechtsmethodik und Software-Engineering“ von der Juristischen Fakultät der Ludwig-Maximilians-Universität München als Seminararbeit angenommen. Literatur konnte bis zum Oktober 2018 berücksichtigt werden. Mein Dank gebühren Herrn Prof. Dr. Hans Christoph Grigoleit (Ludwig-Maximilians-Universität München) und Herrn Prof. Dr. Florian Matthes (Technische Universität München).

* Bacharel em Direito pela Faculdade de Direito do Largo de São Francisco, com Zertifikatsstudium pela Ludwig-Maximilians-Universität München (LMU), e cursando Licence en Droit pela Université Lyon III. 
propriamente jurídica contida sob o que tecnicamente se costuma denominar smart contracts, a qual será, nessa interface do direito com a tecnologia, referida como smart legal contracts. Nesse tom, analisar-se-ão: (i) seu enquadramento legal; e (ii) suas possíveis aplicações frente ao universo jurídico alemão, em especial à seara contratual. Essa investigação justifica-se, sobretudo, pelo potencial modificador dos referidos "contratos" na dinâmica do segmento - dentre possíveis modificações, é possível mencionar a simplificação e a automatização de processos, por exemplo, propiciados pelo seu inerente mecanismo de autoexecução.

Palavras-chave: Smart Legal Contracts. Contratos. Programas automáticos. Blockchain. Autoexecutabilidade.

\begin{abstract}
:
The scope of this paper is to study, from a legal perspective, the recent figure of smart contracts, automatic software programs built on blockchain platforms. More specifically, it is studied how they may impact the contractual legal world, acting as the Law's response to the challenges proposed by the society, that is permanently evolving. Basically, it will be investigated whether these contracts are, effectively, an innovative legal tool or not - an issue that, considering their incipient disposition, has not yet been resolved. It is expected to clarify the actual legal side of what is technically known as smart contracts, which will be referred, in the interface of law and technology, as smart legal contracts. Thus, it will be analyzed: (i) its legal framework; and (ii) its possible applications vis-à-vis the German legal world, particularly in the contractual aspect. This investigation is justified, above all, by the potential of the referred "contracts" of modifying the segment dynamics - among possible modifications, it can be mentioned, for instance, the simplification and automation of processes provided by its inherent self-execution mechanism.
\end{abstract}

Keywords: Smart Legal Contracts. Contracts. Automatic programs. Blockchain. Self-executability.

\title{
I. Darstellung des Problems
}

Durch die Entstehung technologischer Innovationen in Bereichen wie Informationstechnologie und Kommunikationsmedien werden Aspekte der Realität dynamischer. Es ist ein Prozess unbestreitbarer, progressiver Tendenz, physische Unterstützung in elektronische Daten umzuwandeln, um den der heutigen Gesellschaft innewohnenden Bedürfnissen fortschrittlicher Komplexität besser Rechnung tragen zu können. Die Entwicklung der Technologien wird im wesentlichen durch die Popularisierung des globalen Computernetzwerks vorangetrieben, welches dazu beigetragen hat, dass die Gesellschaft progressiv in eine Phase der Umwandlung zur digitalisierten Welt eintrat.

Als Konsequenz erregt diese nachweisbare Tendenz eine Reaktion von seiten der Welt des Rechts, das von vielen als innovationsfeindlich angesehen wird. Daher entsteht eine Debatte zur Position des Rechts und Rechtsinhabers gegenüber den modernen Technologien. Nach M. G. Losano könne der Jurist Recht als nicht reduzierbar 
gegebene Größe verstehen und somit als Formatierer aufkommender Techniken, oder Recht als Ergebnis einer bestimmten sozioökonomischen Realität begreifen, aus welcher sich für den Betreiber die Aufgabe ableiten würde, das juristische Universum auf eine gewisse Formbarkeit hin zu überprüfen, welche fähig sein müsse, im Laufe der Zeit neue Techniken zu absorbieren. Hierbei geht es vor allem um zwei mögliche Haltungen, welche sich nicht ausschließen, wohl aber ergänzen (LOSANO, 1974, p. 2-4).

Bei Prüfung des Rechts nach dieser zweiten Betrachtungsweise gestaltet sich notwendige Haltung nicht normativem Positivismus untergeordnet dem juristischen Denken verinnerlicht (FARIA, 2016, p. 273). Es geht um die Grundlage für den Erfolg aktueller untersuchender Analyse, welche die Thematik nicht isoliert aus dem Zusammenhang technologischer, ökonomischer und sozialer Transformationen untersucht, sondern selbige als Phänomen, eine Gruppe juristischer Gegebenheiten betrachtet.

In diesem Sinne lässt der progressive Fortschritt auch im juristischen Milieu vielversprechende Werkzeuge von anlagen verschiedener Segmente erscheinen. Konkret lässt sich das oben bereits von M. G. Losano beschriebene Aufeinandertreffen verifizieren.

Dem juristischen Universum Formbarkeit zum Absorbieren neuer Techniken zuzusprechen sei daher der relative Schlüssel des Rechts zur Überwachung der neuen- und ständigen Bedürfnisse, welche Bühne der heutigen Realität sind. Noch vor dem Rechtsinhaber an sich beweist die Geschichte, dass neue Lösungen über die Person des Unternehmers gesucht werden, welche in den Schumpeterschen Diktaten eine Innovation als Form der Gleichgewichtsverschiebung zur konsequenten ökonomischen Entwicklung innerhalb des bekannten Prozesses der kreativen Zerstörung des Kapitalismus anstrebt (SCHUMPETER, 1975, p. 82-85). Noch vor faktischer Notwendigkeit erscheint die imperative Konfrontation solcher durch die Rechtsinhaber.

In Bezug auf das Thema vorliegender Studie ist der Ausblick nicht verschieden. Angesichts gegenwärtiger Situation, in welcher es latente, dynamische Impulse als externes Element des Rechts gibt, möglicherweise ausgestattet vom transformierenden Element des modus operandi in juristischer Tradition, ist es Sache des Jura Studenten aufmerksam gegenüber möglichen Transformationen zu sein. Diese in Frage stellende Einstellung ist essenziell, damit es keine unerwünschte Abgrenzung zur juridischen Mission, nämlich deren Dienst an der Gesellschaft, gibt.

In genau diesem Zusammenhang sei vorliegende Arbeit entwickelt. Eingebettet in einem revolutionierenden Kontext fortschrittlicher Technologien in Materien wie Sicherheit, Wahrung von Rechten, Privatsphäre und Inklusion (TAPSCOTT; TAPSCOTT, 2016, p. 61-84) ist das Netzwerk von Transaktionen und Daten genannt Blockchain, deren besondere Eigenschaften Transparenz, die Unveränderlichkeit des Betriebsregisters und das Vorherrschen von Konsensregeln sind. Dieses Netzwerk 
exponentiellen Wachstums in Bereichen der modernen Wirtschaft hat in seinen KryptoWährungen wie Bitcoin seine bekannteste Manifestation.

Da es sich jedoch um ein System handelt, das in der Lage ist, eine große Vielfalt von Transaktionsinformationen zu beherrschen, vertritt die BlockchainTechnologie ein direktes Anwendungspotenzial viel größerer Dimension als der monetäre Bereich. Dabei können ihre Applikationen auch klare juristische Konsequenzen haben, nachgewiesen in ihrer Ermittlungstätigkeit zugewandt dem vertraglichen Bereich: ${ }^{2}$ Die sogenannten Smart Legal Contracts.

Im Allgemeinen ist der Ausdruck Smart Legal Contract als Gattung des Smart Contract Genres konfiguriert. Dieses wiederum besteht aus automatischen Software Programmen, die auf einem Blockchain Protokoll aufgebaut sind. Praktisch gesehen sind sie in der Lage, zu überprüfen, ob bestimmte Bedingungen, die durch die Vertragsparteien vorher vereinbart wurden, erfüllt sind und dann selbstständig die vorher programmierte Konsequenz eigenständig durchführt. Rechtlich wiederum ist das Festlegen einer vertraglichen Vereinbarung möglich, gefolgt von der Vereinbarung und Errichtung vertraglicher Normen beteiligter Parteien. Bei kontrolliertem Befolgen dieser obligatorischen Beziehung zwischen den Vertragsparteien treten die von vornherein verdeutlichten Bedingungen des Rechtsgeschäfts in Kraft. Sie garantieren Unveränderlichkeit, Endgültigkeit und Transparenz. Man beachte, dass der Terminus Smart Contract in seinem Wesen nicht ein Juristischer ist. Es kann sich aber andererseits (und hierbei betreten wir das Universum des Rechts) eine Verpflichtung zwischen verschiedenen Parteien bilden. Dies ist die Komplexität des Themas, welche eine nicht nur juristische-, sondern auch technologische Analyse hervorruft und zwingend notwendig macht, notwendigerweise über Voraussetzungen und das Funktionieren der BlockchainTechnologie.

Die Geschichte lehrt, dass sozioökonomische Entwicklungen Vertragsrecht kategorisch und dringlich für die Gesellschaft machen (ATIYAH, 1981, p. 3). Inmitten des heutigen von Innovationen durchdrungenen Universums kann dieses Gesetz nicht statisch bleiben. Und in diesem Moment möglicher Eignung neuer Trends setzt gegenwärtige Untersuchung an: Möglicherweise können sich Smart Legal Contracts innerhalb des vertraglichen Systems positionieren als ein Schritt vorwärts auf dem komplexen und qualvollen Pfad rechtlicher Entwicklung und Verbesserung.

Vgl. Breidenbach; Glatz (2018, p. 116). 


\section{Blockchain-Technologie}

Die Studie zur Blockchain-Technologie erfährt ihre Rechtfertigung, indem sie geltend macht, es werden über diese Protokolle Anwendungen wie die Smart Contracts (sowie für das Recht entsprechende Smart Legal Contracts) betrieben. Die juristische Konzeption und das Untersuchen der Anwendbarkeit der Smart Legal Contracts auf dem Gebiet des deutschen Vertragsrechts fordern als Voraussetzung Klarheit über technologische Aspekte betreffend Blockchain-Technologie, jenem Netzwerk, in welchem solche Verträge entworfen und registriert werden.

\section{Konzept}

Blockchain ist der Name einer Technologie bestehend aus einer Bank verteilter Daten, die alle betreffenden Datensätze zu laufenden Transaktionen speichert, die von ihren Teilnehmern ausgeführt werden. Es geht hierbei um ein peer-to-peer Netzwerk (P2P), ${ }^{3}$ welches notwendigerweise durch Dezentralisierung bei der Datenvalidierung- und Lagerung gekennzeichnet ist.

Mit dem Aufkommen dieser offenen Netzwerktechnologie, das Ergebnis jahrzehntelanger Forschung Verschlüsselungstechnik, ist es Internetnutzern erstmals möglich, physische, immaterielle und digitale Werte sicher und garantiert an einen anderen Nutzer zu übertragen. Die Werbung und Legitimität von Transfer durch Blockchain auf analoge Weise zu einem von den Benutzern des Netzwerks geteilten kollektiven Grundbuch, das unveränderbar ist und Transaktionen aufzeichnet, sind es, was den innovativen Charakter der Technologie ausmacht. Größe ist die von der BlockchainTechnologie gebrachte Revolution, welche prominente Venture Investoren mit dem Effekt der erschienenen PCs der siebziger Jahre, oder aber mit dem Einzug des Internets Mitte der neunziger Jahre vergleichen, wenn sie die möglichen Auswirkungen der BlockchainTechnologie untersuchen (WRIGHT; DE FILIPPI, 2015, p. 2).

Diese Koordination individueller Aktionen als Geschenk der BlockchainTechnologie, gesteuert von einem dezentralisierten Konsensmechanismus, ist etwas völlig neuartiges. Früher, wie auch im Internet selbst, war eine solche Anstrengung nur mittels zentralem Organs möglich, welches die Gültigkeit einer bestimmten Transaktion sicherte. Gerade aus diesem Potential der Dezentralisierung, ${ }^{4}$ sei es bei der Datenspeicherung, sei es beim Informationsmanagement und mit der Absicht, die Rolle des zwischengeschalteten

\footnotetext{
3 „These [P2P transactions] are decentralized transactions that involve only the two parties (...). There are no third-party intermediaries (such as a bank) involved in peer-to-peer transaction“" (LEE, 2016, p. 131).

$4 \quad$ Vgl. P. Quiel (2018, p. 567).
} 
Regulators zu verringern, entwickelt sich diese Technologie mit der Kapazität, technische Grundlagen bereitzustellen zum Schaffen dezentralisierter Währungen, neuer und demokratischerer Regierungssysteme, dezentralisierter Organisationen befähigt via Computernetzwerke ohne menschliches Zutun arbeiten zu können (WRIGHT; DE FILIPPI, 2015, p. 1) zusätzlich zum Schwerpunkt aktueller Forschungstätigkeit: Smart (Legal) Contracts.

Im Hinblick auf das Konzept der Blockchain-Technologie wird im Allgemeinen das besondere Merkmal der Ähnlichkeit zwischen dem Netzwerk und einem verteilten Grundbuch hervorgehoben. Diese Verteilung von Informationen im Netzwerk ist unerlässlich, um die Unabhängigkeit vor Organen und Dritten Zentralisierern zu gewährleisten, die bei der Validierung von Transaktionen tätig sind. Mit solchen grundlegenden Aspekten stellt sich Blockchain ansonsten als eine Technologie vor, die mit einer extrem technischen, komplexen und ausfallsicheren Konfiguration ausgestattet ist (PAECH, 2016, p. 638).

Obwohl die Blockchain gemeinsamen Ursprung mit berühmter Bitcoin hat, gibt es heute andere Blockchain-Netzwerke. In Bezug auf die Blockchain des Ethereums wird bestätigt, dass es sich um eine Blockchain handelt, die nach der Blockchain des Bitcoins entstanden ist. Ähnlich geht es auch hier um ein Open-Source-Netzwerk. Das Ethereum-Netzwerk stellt sich jedoch unverwechselbar als praktikable Plattform für ein alternatives Protokoll vor, dass Entwurf und Ausführung von dezentralisierten Anwendungen wie etwa Smart Contracts und Dapps (dezentrale Anwendungen) ermöglicht. (BUTERIN, 2014, p. 9).

Daher liegt die Bedeutung der Ethereum-Plattform (in der die Einheit Ether - ETH, so, wie in Bitcoins Blockchain die Einheit BTC liegt) für Zwecke vorliegender Untersuchung gerade in ihrem höheren erfinderischen Charakter. Wie man sieht, ermöglicht dies die Schaffung von mehreren dezentralisierten Anwendungen auf eine praktischere und diversifiziertere Art und Weise als im Vergleich zu dem Blockchain-Netzwerk, welches ursprünglich von Bitcoin eingeführt wurde..$^{5}$ Es liegt an der im EthereumNetzwerkprotokoll enthaltenen Intelligenz, die üblicherweise als programmierbare Blockchain bezeichnet wird. ${ }^{6}$

Vgl. Breidenbach; Glatz (2018, p. 115).

$6 \quad$ Vgl Peters; Panayi (2015, p. 7). 


\section{Einsatzmöglichkeiten}

Die Blockchain erscheint heute vielversprechend in den unterschiedlichsten Bereichen $^{7}$ und hat konkret Anwendungen für Felder wie Unternehmen, Finanzen, Banken, Regierungen und auch das Recht. Bei Letzteren heben sich grundsätzlich Smart Contracts als Smart Legal Contracts hervor, die im nächsten Kapitel genauer untersucht werden.

GeradeausdemPotentialderDezentralisierung, seiesbeiderDatenspeicherung (ANTONOPOULOS, 2014, p. 159), sei es beim Informationsmanagement, mit dem Ziel, die Rolle des Intermediärregulators zu verringern, erscheint diese Technologie fähig, technische und wirtschaftliche Grundlagen für ihre Verwendung in unzähligen Situationen zu liefern.

Kurz untersucht sei hier diese Vielfalt von Einsatzmöglichkeiten, ihre Nützlichkeit in Bereichen wie: (i) Geld (dezentralisierte Währungen); (ii) Finanzen (wie Handels- und Wertpapierhandelsanträge und Derivatgeschäfte); (iii) Geschäfte (z. B. Abbildung geschäftlicher Produktionsketten und Dokumentenauthentifizierung); und (iv) öffentliche Verzeichnisse (sei es über Immobilientitel und nicht greifbare Werte wie Patente und Marken, seien es über Abstimmungen und Identitäten). ${ }^{8}$

Deshalb können wir bemerken, wie umfangreich das Studium der möglichen Anwendbarkeiten auf der Basis von Blockchain ist, ${ }^{9}$ und es gibt heute ein ganzes Universum von Lösungen in Entwicklung. Blockchain kann letztendlich als eine Gelegenheit angesehen werden, mehr Transparenz in Beziehungen einzuführen, sei es zwischen Menschen, Unternehmen oder Regierungen.

\section{Smart Contracts latu sensu}

Der Begriff Smart Contract steht nicht unbedingt immer für Verträge unter dem Gesichtspunkt des Rechts. Wie im Folgenden untersucht handelt es sich um ein technisches- und nicht rechtliches Konzept: Smart Contracts sind automatische Softwareprogramme, die auf einem Blockchain-Protokoll aufbauen. Dies bedeutet aus praktischer Sicht, dass solche Programme in der Lage sind zu überprüfen, dass eine bestimmte, von den Parteien vorher festgelegte Bedingung erfüllt wurde und damit ihre programmierte Konsequenz selbstständig auszuführen (ähnlich einer Bedingung ,wenn A, dann B“').

\footnotetext{
$7 \quad$ Siehe Wiefling; Lo Iacono; Sandbrink (2017, p. 483 et seq).

8 Über Fragen wie die Beziehung zwischen Blockchain Technologie und Datenschutzrecht, siehe Quiel (2018, p. 566 et seq).

9 In diesem Sinne, siehe McLean; Deane-Johns (2016, p. 97-102).
} 
In diesem Sinne wird aufgrund der breiten Brauchbarkeit, welche die Smart Contracts bringen das von R. Kadamani, einer brasilianischen Rechtsanwältin und Blockchain-Expertin, vorgeschlagene Verständnis und Klassifizierung verstärkt, wonach es angesichts dieser automatischen Softwareprogramme drei mögliche Szenarien gibt: (i) rechtsgültige Verträge ohne Smart Contracts; (ii) Smart Contracts, bei denen es sich nicht um Verträge im juristischen Sinne handelt und (iii) Die Kreuzung zwischen rechtsgültigen Verträgen und Smart Contracts (KADAMANI, 2018).

\section{Smart Contracts}

In der folgenden Studie sollen das Konzept und die Funktionsweise dieser Softwareprogramme untersucht werden, ohne dass es irgendwelche Verbindungen mit dem Rechtsuniversum gibt. Die Untersuchung erklärt sich aufgrund der noch immer fremden Natur der Rechtsdisziplin angesichts der Entstehung und schnellen Entwicklung dieser Technologien: Es ist nicht durchführbar, Smart Contracts zu prüfen, die auf Anwendungen wie Verträge im juristischen Sinne (die sogenannten Smart Legal Contracts) abzielen, ohne Verständnis über die Technologie und ihre wesentlichen Konzepte.

Anfänglich wurde das Konzept und der Begriff Smart Contract von N. Szabo (SZABO, 1997), einem nordamerikanischen Juristen, Kryptologen und Informatiker bereits in den 1990er Jahren konzipiert. Für Szabo wären Smart Contracts im Großen und Ganzen wie Transaktionsprotokolle von Computern, die die Bedingungen eines Vertrags so umsetzen, dass sein Bruch kostspielig, wenn nicht gar prohibitiv wird (SZABO, 2002). In diesem Sinne dachte Szabo an Vertragsklauseln wie etwa Garantie, Verbindlichkeit und Abgrenzung von Eigentumsrechten und erwog die Möglichkeit, diese in sowohl Hardware und Software einzuarbeiten, um zunächst Komplikationen zu fördern für selbiger Abbruch (SZABO, 2002).

Weiterhin hatte der Autor das Verständnis, diese Programme nicht nur dazu zu nutzen, um bestimmte Klauseln als Werkzeug einzusetzen, sondern auch Kryptographie arbeiten zu lassen und damit Schutz vor Betrug und Verstößen zu garantieren sowie Transparenz im gesamten System zu gewährleisten. Im Allgemeinen ginge es um Programme, die zu einer Senkung der Kosten für Vermittlung, Schiedsverfahren und selfenforcement führen würden (SZABO, 2002).

Wie man sieht, bestehen Smart Contracts aus Computerprogrammen, welche in der Lage sind, Ausführung geplanter Konsequenzen zu automatisieren (RASKIN, 2017, p. 309). Mit anderen Worten sind sie programmierbare Codes, die den Inhalt einer bestimmten Beziehung haben, die Vorgänger und nachfolgende einbeziehen durch eine Reihe von Verfahrensanweisungen festgelegt, und die Protokolle mit 
Benutzerschnittstellen kombinieren, um diese Beziehungen in dem Computernetzwerk zu formalisieren und zu sichern (SZABO, 1997).

Konkret arbeiten sie elektronisch und sind aus Codes zusammengesetzt (in der Programmiersprache Solidity, z. B. wenn auf der Plattform Ethereum geschrieben), die die Bedingungen (Vertragsklauseln) und die vorhergesagten Ergebnisse genau spezifizieren indem sie dem Programm einverleiben.

Ursprünglich verzichtete dieses grundlegende technische Konzept von Smart Contracts (Automation und automatische Exekution) auf das BlockchainNetzwerk. ${ }^{10}$ Jedoch mit dem Gebrauch von dezentraler Peer-to-Peer-Technologie gibt es eine Ermächtigung solcher Codes in dem Maße, ${ }^{11}$ dass größere Transparenz und Sicherheit möglich ist, und darüber hinaus natürlich Dezentralisierung bei der Validierung und Speicherung von Informationen zur die Ausführung dieser Codes. ${ }^{12}$ Transparenz hier, im Sinne von Überprüfbarkeit, das heißt, zu wissen, welches Regelwerk den Vertrag regelt und welche Informationen die andere Partei speichert. Es unterscheidet sich daher beispielsweise von Programmen in Python, ${ }^{13}$ in denen es nicht möglich ist, den auf einem anderen Computer ausgeführten Code auf eine breite und schnelle Art und Weise zu überprüfen. In den Smart Contract Systemen von Blockchain gibt es eine vollständige Isolierung und Offenlegung des Codes, wodurch der gesamte Prozess von allen verifizierbar sei.

Dezentralisierung ihrerseits steht in Beziehung zur Garantie von Integrität und Anwendbarkeit des Vertrags. Dies liegt daran, dass es in Fällen, in denen das Programm in den Händen einer einzelnen Einheit liegt, natürlich einfacher für sie ist, seine Bedingungen zu ändern, oder sogar einseitig seine Ausführung zu vermeiden. Als Antwort hierfür erlaubt die Dezentralisierung eine unabhängige und netzwerkweite Verifikation, die es erschwert, Betrug zu begehen.

Basierend auf der konkreten Möglichkeit einer sicheren, transparenten und dezentralen Umgebung wird dieses Potenzial von Blockchain-Netzwerken als wesentliches Instrument für die Umsetzung, Anerkennung und Nutzung von Smart Contracts gesehen.

Das ist die Erklärung für die bemerkenswerte und wachsende Attraktivität von selbst-ausführenden Programmen, die mit der Blockchain-Technologie in Korrelation stehen.

10 Zur konzeptionellen Unabhängigkeit in Bezug auf Smart Legal Contracts und dem dezentralen Blockchain Netzwerk, siehe Fries (2018b, p. 86).

11 Vgl. Kaulartz; Heckmann (2016, p. 138-140).

12 Vgl. Breidenbach; Glatz (2018, p. 114).

13 Python ist der Name einer Programmiersprache, deren Ziel es ist, eine Sprache mit hoher Produktivität und Lesbarkeit zu sein. 
Darüberhinaus ist wichtig, hervorzuheben, dass diese Programme nur dann selbst ausgeführt werden, im Falle, dass sich der Aktive in Bezug auf ihre Verwaltung und Ausführung im Blockchain-Netzwerk befindet (SONG, 2018). Diese Einschränkung bezeichnet daher das aktuelle Limit Smart Contracts-Aktivitäten: Wenn sie isoliert verwendet werden, ist es nicht möglich, Programme selbst auszuführen, deren Assets (oder Tokenisierte Assets ${ }^{14}$ ) nicht im Blockchain-Netzwerk repräsentiert sind.

Die Implementierung eines Smart Contract on-chain, die über verifizierte Vorkommnisse kommunizieren und sich auf in Blockchain repräsentierte Assets beziehen, ist keine komplizierte Aufgabe. Wenn es jedoch für einen Smart Contract notwendig ist, mit der physischen Welt zu interagieren, erfordert dies die Verbindung zu einem Oracle. Dies sind diejenigen, die das Programm über das Auftreten oder Nichtvorhandensein einer bestimmten Bedingung informieren und in diesem Fall die Ethereum-Plattform mit der Außenwelt verbinden (off-chain) (SONG, 2018). Dadurch wird dem Code erlaubt, externe Daten in das Netzwerk importieren.

Schließlich verdient die Bezeichnung von Begriffen wie Smart Contracts einige Worte. Es ist eine unangemessene Nomenklatur, die, indem sie die Wörter „Smart“ und „Contract“ enthält, den Leser leicht dazu bringt, sie auf eine ungeeignete Art zu interpretieren. ${ }^{15}$

Smart Contracts sind nicht mit Elementen ausgestattet, die sie ,intelligent“ machen (KAULARTZ; HECKMANN, 2016, p. 618 et seq). Sie beziehen sich lediglich ganz einfach auf ein technisches Konzept für die Koordination zwischen Vorgängern und vorherbestimmten Nachfolgern, das in eine Logik der Selbstausführung eingefügt ist (MAINELLI; MILNE, 2016, p. 79). Dies, verbündet mit Blockchain-Netzwerken und der Fähigkeit, sie für die Unterbringung solcher Programme zu programmieren, garantiert minimierte Transaktionskosten und reduziert Risiken auf transparente und dezentrale Weise.

Gerade weil sich Smart Contracts in diese selbstausführenden Rechencodes übersetzen mit dem Ziel, Operationen zu schützen und auszuführen (wesentlich in Blockchain), gibt es diejenigen, die sagen, der Begriff Autonome Agenten sei vorzuziehen, zum Zweck solche Programme zu qualifizieren. ${ }^{16}$

14 Tokenisierte Assets sind Offline-Assets, die durch digitale Assets repräsentiert werden. Im Falle von Smart Contracts ist es daher nicht notwendig, dass der zugeteilte Vermögenswert notwendigerweise eine KryptoMünze sei, wie zum Beispiel Ethereum. Es reicht bereits, dass es sich um ein digital repräsentiertes Asset handelt (Digital Token). Vgl. Catchlove (2017, p. 7).

15 Vgl. Breidenbach; Glatz (2018, p. 115).

16 Die Terminologie „Autonome Agenten“ in Bezug auf Smart Contracts ist zum ersten Mal verwendet, sogar in Ethereums eigenem White Paper, das ist ein Dokument, in dem das gesamte technische Konzept und die Funktionsweise des Netzwerks aufgeführt werden. Vgl. Buterin (2014). 
Es ist zwingend, dass in Bezug auf den Begriff „Vertrag“ unbedingt zwischen den möglichen Szenarien, die durch Smart Contracts abgedeckt werden können, unterschieden wird. Die Nomenklatur ist daher dem Recht völlig fremd. ${ }^{17}$ Sie kann sich jedoch in einigen Fällen auf das Rechtsuniversum beziehen, indem sie analog zu den Verträgen in ihrem juristischen Sinne funktioniert - den sogenannten Smart Legal Contracts.

\section{Smart Legal Contracts \\ 2.1. Konzept}

Bei Smart Legal Contracts erfolgt die vertragliche Verpflichtung nach folgender Vorgehensweise: Zunächst einmal werden geeignete Vertragsklauseln in einen rechnerisch ausführbaren Code umgewandelt (RASKIN, 2017, p. 309) und in einer zweiten Phase im Kollektivnetz registriert; in dritter Phase, mit dem Antreffen der vorbestimmten Bedingung (PERUGINI; CHECCO, 2016, p. 11) oder mit der Verletzung von vertraglichen Verpflichtungen, beide Szenarien mathematisch bestimmbar, wird das Ereignis produziert, was digital verbunden war. ${ }^{18}$

Dies beweist eine synonyme Unterscheidung zwischen $(i)$ möglichem Nutzen und Vereinfachung vertraglicher Praktiken, wo jeder Anwender einen Vertrag erstellen kann, indem er einfach eine Transaktion in der Blockchain registriert (DELMOLINO; ARNETT; KOSBA; MILLER; SHI, 2015, p. 4) und; (ii) es beweist Verbesserung der Effizienz und Leistung im Rahmen des Vertragsrechts, da sie die Rolle des Dritten ersetzt, der normalerweise zur Beilegung von Meinungsverschiedenheiten erforderlich ist (LEE, 2016, p. 113).

Aus den von Blockchain gebotenen Qualitäten der Transparenz, der Unveränderlichkeit und der Publizität wären die Verträge, die in das gemeinsame und kollektive Netzwerk dieser Technologie eingegangen sind, damit ausgestattet, dass die Parteien individuellen Deklarationen in konkreten Situationen zuzustimmen könnten und eine solche Verpflichtung auf sichere und verifizierbare Weise registrieren (WRIGHT; DE FILIPPI, 2015, p. 1). ${ }^{19}$

In solchen Verträgen, wie hier kurz vorweggenommen, gelten die Informationen als self-enforceable zum Zeitpunkt ihres Einsetzens in das dezentralisierte Netz, was rechtliche und funktionale Probleme mit sich bringt ihren Betrieb entsprechend.

\footnotetext{
17 Über die terminologische Ungenauigkeit des Begriffs Smart Contract zur Beschäftigung im juristischen Bereich, siehe Schrey; Thalhofer (2017, p. 1.431).

18 Vgl. Fries (2018b, p. 86). Siehe auch Kaulartz; Heckmann (2016, p. 618).

19 Siehe Heckmann; Schmid (2017, p. 13 et seq).
} 
Vereinfachung, Automatisierung der Prozesse, Erhöhung der Standardisierung (standardisation), Angebot der elektronischen Ausführung, Versorgung mit sofortiger Integration von Verträgen in Datennetzen und die bereits erwähnte Selbstausführung (ROUGHTON; BIDEWELL, 2017, p. 3) sind Eigenschaften, die a priori an den Smart Legal Contracts ins Auge fallen.

Durch das Konsens-Protokoll der dezentralen Netzwerktechnologie gibt es eine Validierung durch das kollektive Netzwerk und das Inkrafttreten der in dem Sachplan programmierten vertraglichen Informationen, was mehr Sicherheit schafft, weil, sobald ein solcher Prozess durchgeführt wurde wurde, selbiger nicht mehr geändert werden kann.

Unter Smart Legal Contracts werden heute im Allgemeinen computergestützte und digitale Verträge verstanden, bei denen Performance und Enforcement der Vertragsbedingungen automatisch und ohne menschliches Eingreifen erfolgen (RASKIN, 2017, p. 322). In dieser Logik liegt die folgende instrumentelle Beziehung: Während Standardverträge die Bedingungen einer verpflichtenden Beziehung zwischen den Parteien beschreiben, verpflichten und sichern Smart Contracts mittels Codezeilen die Selbstausführung dieser Verpflichtungen.

Auf der einen Seite bezieht sich die Effizienz, die in den vertraglichen Umfang eingeführt werden kann, auf die Prüfung, Ausführung und Platzierung in Kraft der Bedingungen des bilateralen Rechtsgeschäfts. Gleichzeitig garantiert sie Unveränderbarkeit, Unwiderruflichkeit und Transparenz. So wird in Vertragsmaterie eine Minimierung der Verhandlungs- und Transaktionskosten garantiert.

Auf der anderen Seite zeichnet sich noch das Potenzial, dass rechtliche Smart Contracts zugunsten eines höheren Sicherheitsniveaus für die Partner in Bezug auf die verwendete Sprache sprechen aus. In solchen Verträgen werden häufig auftretende rechtliche Probleme wie Fehler in der Interpretation und Verständnislosigkeit, die auf den oft mehrdeutigen Wortlaut von Klauseln im vertraglichen Instrument zurückzuführen sind (CATCHLOVE, 2017, p. 15), zu großem Teil vermieden. ${ }^{20}$ Dies erklärt sich dadurch, dass während traditionelle Verträge die Rechtssprache für ihr Exposure verwenden, Smart Legal Contracts jedoch, wie untersucht, auf Aussagen der Natur ,wenn A, dann B“ basieren. Aussagen, deren Inhalt einen geringeren Grad an Zweifelhaftigkeit enthält (CATCHLOVE, 2017, p. 15).

Es bestätigt sich damit die Fähigkeit der Smart Legal Contracts, um die Zuverlässigkeit von Transaktionen und die Ausführung von Verträgen auf eindeutige Weise zu garantieren, ohne dass es ein Eingreifen des Menschen gäbe oder selbiges erforderlich

20 Aufgrund der Beschränkung der Programmiersprache im Gegensatz zur traditionellen Rechtssprache gibt es immer noch Spielraum für Mehrdeutigkeiten. Diese sind jedoch viel weniger vorhanden. Vgl. Catchlove (2017). In diesem Sinne, siehe auch Raskin (2017, p. 324). 
wäre. Ihre Anwendbarkeit entspricht letztlich der klaren Erfüllung vertraglicher und wirtschaftlicher Kriterien von Effizienz (RASKIN, 2017, p. 336), die die Kapazität des Rechts als Lösungsanbieter für die verschiedensten heutigen Themen stärken.

Bei der Untersuchung der wesentlichen Eigenschaften, die in einem Smart Legal Contract vorhanden sein müssen, und im Einklang mit der Studie von S. Bourque und S. Fung Ling Tsui, ${ }^{21}$ fällt Folgendes auf: (i) angebrachte Identifizierung der Parteien und Zustimmung unter ihnen zu den Vertragsbedingungen; (ii) digitale Identifizierung juristischer Smart Contracts und die Möglichkeit des Zugangs zu ihnen über das dezentrale Netz; (iii) freier Zugang der Parteien zu den Bedingungen und weiteren Informationen des Vertrags (Informationen über die Parteien, Vertragsstatus, Ereignishistorie usw.); (iv) vertragliche Klarheit, die Mehrdeutigkeiten bereinigt, und Programmiersprache (auch wenn Smart Legal Contracts mit nicht automatischen Klauseln existieren - Präambeln zum Beispiel); (v) Vorhandensein von Sprachen in der Art wie ,wenn A, dann B“, die die Automatisierung von Smart Legal Contracts erlauben; (vi) Speichern der Historie von Geschehnissen, aus denen die vereinbarten Vertragsfunktionen ausgeführt werden können.

\subsection{Rechtliche Rahmenbedingungen}

De lege lata, die aktuelle Position von Smart Legal Contracts wird vor der deutschen Vertragsperspektive untersucht, d.h. welche juristische Behandlung dieser neuen Realität im Rahmen von Verträgen zukommt.

Das Rechtsmodell der Vertragsbildung wird durch eine Reihe von Auffassungen und Regeln abgegrenzt, die sich wie alle juristischen Regeln zur Durchführung bestimmter praktischer Ziele und damit zum Dienen bestimmter Interessen eignen. ${ }^{22}$ In diesem Sinne scheint es angebracht, eine kurze Analyse der Vertragsphasen in den Smart Legal Contracts durchzuführen, zum Zwecke der Überprüfung, ob selbige den Rechtsbegriffen deutscher Rechtsordnung entsprechen oder nicht.

Was die vertragliche Formation angeht und insofern, als der Vertrag notwendigerweise ein bilaterales Rechtsgeschäft ist, muss es mindestens zwei Teile und damit zwei Willensäusserungen geben (AUNERT-MICUS; GÜLLEMANN; STRECKEL; TONNER; WIESE, 2018, p. 93 et seq). Diese Parteien äußern ihren Wunsch, als Konsequenz der Gesamtheit der Vertragsklauseln, an eine gemeinsame Regelung ihres gegenseitigen Verhältnisses gebunden zu sein (ROPPO, 2009, p. 72).

$21 \quad$ Siehe Bourque; Fung Ling Tsui (2014).

22 Vgl. Roppo (2009, p. 93). 
Bei der Prüfung der $\S \S 145$ und 147 des Bürgerlichen Gesetzbuchs (BGB), fundamentale Rechtstexte zur Prüfung der Vertragsgestaltung, stellt man fest, dass die Verträge sich aus Inhalten bzw. aus Antrag und Annahme bilden (SCHREY; THALHOFER, 2017, p. 1.431). Wie bei allen anderen Verträgen ist es auch bei Smart Legal Contracts erforderlich, dass es einen Vorschlag gäbe von einer der Parteien kommend, gefolgt vom Akzeptieren der anderen Partei. Und genau in diesem Treffen der Willen, wie auch in diesen Verträgen (HECKELMANN, 2018, p. 505), ist die vertragliche Bindung verbindlich konzipiert, und sie schafft Rechte und Pflichten für die Parteien (ROPPO, 2009, p. 72).

Was das vertragliche Angebot betrifft, so ist die Affinität zwischen dem, was in traditionellen Verträgen geschieht, und dem Szenario der Smart Legal Contracts klar (RASKIN, 2017, p. 322): In beiden Verträgen gibt es den Vorschlag und das Akzeptieren.

$\mathrm{Zu}$ einem späteren Zeitpunkt muss der das Angebot der anderen Partei Akzeptierende (Welches als Resultat das Manifestieren eines Vertrags bzw Smart Legal Contract hat) lediglich das im Netzwerk repräsentierte Eigentum (,tokenisiert“) in den Code transferieren (CATCHLOVE, 2017, p. 11). Der Vertrag findet also statt, weil ein solches Verhalten in den Smart Legal Contracts gerade das Kommunizieren des Akzeptierens bereitstellt (CATCHLOVE, 2017, p. 11): dies zeigt sich hier durch die Performance (RASKIN, 2017, p. 322). ${ }^{23}$

Die vertragliche Beendigungsphase stellt den Abschluss eines bestimmten Vorgangs dar, der gesetzlich anerkannt und geschützt ist (ROPPO, 2009, p. 211).

Dieses Geschäft wird dann errichtet, wenn die zwischen den Parteien vereinbarten Rechtswirkungen hergestellt werden. In diesem Sinne gibt es wiederum deutliche Ähnlichkeit zwischen dem, was in traditionellen Verträgen und in Smart Legal Contracts geschieht.

Bei letzteren wird ihr Abschluss z. B. durch die Übertragung von zuvor durch die im Vertragscode gesendeten tokenisierten Assets verifiziert.

Im Vergleich zwischen Bildung ${ }^{24}$ und der Vertragsbeendigung ${ }^{25}$ zwischen Smart Legal Contracts und traditionellen Verträgen gibt es keinerlei konzeptionelle

23 Deshalb gibt es den Vergleich zwischen den Smart Contracts (latu sensu) und Warenautomaten. Die Mechanik der Warenautomaten bewirkt, dass das Transferieren automatisch abläuft, und es ist gerade das Verhalten des Kunden des Zahlens, das Ereignis, dass zum Abschluss des Kaufsvertrages mit dem Eigentümer der Maschine führt. Vgl. Kaulartz; Heckmann (2016, p. 621).

24 Davon abgesehen, dass Smart Legal Contracts nichts anderes als automatische Softwareprogramme sind, sind sie nicht einseitig geschaffen. Das heißt, wie bei herkömmlichen Verträgen ist eine Willensvereinbarung zwischen den Vertragsparteien erforderlich.

25 Es ist bei Smart Legal Contracts nicht möglich, etwas zu löschen, wie es in einem automatischen Softwareprogramm vorkommen kann, das heißt ohne, dass sich erwartete Konsequenzen ereignen. In diesem vertraglichen Instrument kann die Kündigung wie bei traditionellen Verträgen bilateral oder einseitig (je nach Fall) erfolgen. In letzterem Fall geschehen jedoch die im Code vorhergesehenen Konsequenzen automatisch, um ein Sich Entziehen der Vereinbarung von seiten der Vertragspartei unmöglich zu machen. 
Elemente, die dem traditionellen juristischen modus operandi (RASKIN, 2017, p. 322) und den Rechtsbegriffen im BGB fremd sind. Smart Legal Contracts registrieren, automatisieren und repräsentieren mehr oder weniger andere Verträge über Blockchain. Gerade deshalb behalten sie die vertraglichen Entstehungs- und Beendigungsmerkmale so, wie sie die Verträge hätten, wenn sie nicht auf der dezentralen Plattform registriert wären. Sie ändern daher keine Vertragsregeln.

Im Wesentlichen wird auch in Smart Legal Contracts das Vertragsverhältnis gebildet, wenn es zwischen Angebot, Akzeptieren und der Absicht, Beziehungen einzugehen, eine Konvergenz gibt. Ein Abschluss ergibt sich seinerseits durch das Einhalten oder Nicht-Einhalten, Gelegenheiten, bei denen es Eintreten in Kraft festgelegter Bedingungen gibt.

Von ihrer Berechnungslogik geleitet agieren Smart Legal Contracts, indem sie die vertraglichen Auswirkungen eines anderen Vertrags automatisieren und somit seine korrekte Verwaltung garantieren. ${ }^{26}$ Es ist daher klar ersichtlich, dass sich die Parteien solche automatischen Softwareprogramme zunutze machen, mit der Absicht, rechtlich anerkannte Verträge abzuschließen.

Dies bedeutet jedoch nicht, dass solche Verträge eine selbständige Vertragsart vertreten oder nicht einmal einen inhaltlichen Vertrag darstellen, wie etwa einen Kaufvertrag, einen Darlehensvertrag, einen Leihvertrag, atypische Verträge u.a.. Der Smart Legal Contract reiht sich nicht in diese Kategorien ein. Es sind insbesondere vertragliche Instrumente, befähigt, wenn nicht eine größere Wahrscheinlichkeit des Befolgens der Vertragsbedingungen beiden Parteien immer garantieren zu können (CATCHLOVE, 2017, p. 9), so doch wenigstens schnelle Entschädigung geschädigter Partei zu garantieren im Falle von Vertragsverletzungen.

In diesem Sinne scheinen sie durch ihre Registrierung im BlockchainNetzwerk eine neue Form der Registrierung und Verkörperung von Vertragsarten, und nicht eine unabhängige Vertragsart nach dem BGB zu vertreten. ${ }^{27}$

Aus Sicht des verpflichtenden Vertragsinhalts betrachtet, ist ein Smart Legal Contract wie ein „Werkzeug“, fähig Verträge nach dem Willen der Parteien auszustatten. ${ }^{28}$ In diesem Sinne und in Übereistimmung mit doktrinärem Verständnis des

26 Administration diese von grundlegender Bedeutung für die Unterstützung zur Vorsorge von Rechtsstreitigkeiten und ihre jeweiligen Kosten. In diesem Sinne weist Fries auf die offenkundige Rolle Legal Smart Contracts bei der Konfliktprävention hin. Vgl. Fries (2018b, p. 90). In diesem Sinne siehe auch Fries (2015, p. 6).

27 Vgl. Süme; Zimprich; Vogt (2018, p. 27).

28 In übereinstimmung mit Heckelmann (2018, p. 508): „Gegenstand von Smart Contracts können sowohl schuldrechtliche als auch dingliche Vereinbarung sein. Schuldrechtlich lassen sich prinzipiell alle Vertragstypen abbilden.“ 
deutschen Rechts ersetzen heute die in einem Smart Legal Contract enthaltenen Codes nicht den klassischen Vertrag, sondern ermöglichen lediglich Maßnahmen, um sie zu verwalten und zu automatisieren. ${ }^{29}$ Smart Legal Contracts bieten einen Mechanismus für die Selbstausführung der vereinbarten Bestimmungen. ${ }^{30}$

Im deutschen Rechtsgebiet ist es völlig möglich, eine vertragliche Vereinbarung zu treffen, gefolgt von der Vereinbarung und der Festlegung vertraglicher Regelungen zwischen beteiligter Parteien entwickelt aus der Verwendung der Smart Legal Contracts. ${ }^{31}$ Theoretisch ist es ausreichend, sie nach den gesetzlichen Anforderungen für beide zu programmieren. ${ }^{32}$ Durch die Bildung eines obligatorischen, bilateralen Rechtsgeschäfts (d.h. eine Willenserklärung zweier Parteien, die sich an bestimmte Handlungen untereinander binden, wie z. B. Zahlungen und mehr), stellt ein Smart Legal Contract einen Vertrag für rechtliche Wirkungsweise dar. ${ }^{33}$ Dieser Vertrag seinerseits kann, wie erwähnt, den verschiedensten Vertragssituationen Rechnung tragen.

Es fällt hier auf die Gestaltungsfreiheit des Rechtsgeschäftes nach $§ 125$ BGB.

Trotz seiner formalen Besonderheit wird ein nach dem Selbstausführungsmechanismus eines Smart Legal Contract strukturierter Vertrag auch weiterhin perfekt als rechtsgültiger Vertrag angesehen. Dies ist gerechtfertigt, ist der wesentliche Inhalt dieser Verträge schließlich nicht geändert. ${ }^{34}$ Um als rechtsgültige Verträge nach deutschem Vertragsrecht, so wie alle Rechtsgeschäfte, angesehen $\mathrm{zu}$ werden, müssen sich diese an bestimmte Kriterien anpassen und selbige erfüllen, so dass ihre Existenz und ihre Gültigkeit von der Rechtsordnung zugelassen werden. Im Falle der Nichteinhaltung dieser Ansprüche würde

${ }_{29}$ Zusätzlich zu den Verträgen erwähnt Fries auch noch Finanzinstrumente, wie dies bei der Anwendbarkeit von Smart Legal Contracts in der Projektfinanzierung und bei Darlehensverträgen der Fall ist. Vgl. Breidenbach; Glatz (2018, p. 124 et seq).

30 Abgesehen davon, dass die in den Smart Legal Contracts vereinbarten Regeln Gültigkeit haben, hebt Fries drei wichtige Einschränkungen hervor. Erstens hat die Strecke, die zum Erreichen des traditionellen Vertrags zurückgelegt wurde, Vorrang vor dem Code. Zweitens kann der Code nur dann ein Rechtsverhältnis zwischen Parteien in dem Maß regeln, in welchem die Parteien nicht durch Gesetzeszwang gehindert sind. Drittens, wenn die Regeln der Software einseitig in den Vertrag aufgenommen wurden, müssen selbige einem allgemeinen Verifizieren der Begriffe und Bedingungen unterzogen werden, in Übereinstimmung mit $\S \S 305$ Abs. 1 e 2 Nr.2, 305c Abs.1, 307, 309 Nr.12, 310 Abs. 1 BGB. Vgl. Fries (2018b, p. 87). Für AGBKontrolle, siehe auch Heckelmann (2018, p. 507-508).

31 „Smart Contracts werden üblicherweise auf der Ebene des Schuldrechts betrachtet“ (HECKELMANN, 2018, p. 508).

32 Betreffend die Programmiersprache, die für die Ausarbeitung von Smart Legal Contracts verwendet wird, Heckmann; Schmid (2017, p. 15): „Denn die Gestaltungs- und Formfreiheit aus der Privatautonomie (Art. 2 Abs. 1 GG, $§ 311$ Abs. 1 BGB) erstreckt sich auch auf die freie Wahl der Vertragssprache.“

33 Zum Beweis des Vertragsabschlusses, Möhlenkamp; Wessel (2018, p. 7): „Der smart contract dürfte in der Regel entweder durch das Tatsachengericht gemäss $§ 371$ a Abs. 1 ZPO wie eine Urkunde oder gemäss $\S$ 371 Abs. 1 ZPO im Wege des Augenscheinbeweises zu würdigen sein, abhängig davon, ob der Softwarecode qualifiziert elektronisch signiert ist.“

34 Dieser Inhalt kann sich beispielsweise auf einen Kaufvertrag ( $§ 433$ BGB), einen Tauschvertrag ( $\$ 480$ BGB) oder gar einen Werkvertrag (§631 BGB) beziehen. 
der Smart Legal Contract nicht mehr die erwartete rechtliche Wirkung produzieren lassen und wäre als nichtig anzusehen. ${ }^{35}$

Angesichts mangelnden Einvernehmens betreffend der Notwendigkeit zusätzlicher Regelung für die völlige Aufnahme der Automatisierung, machbar durch Smart Legal Contracts, wird auf die Kombination derjenigen mit traditionellen schriftlichen Verträgen hingewiesen als eine der plausibelsten Optionen auf kurze Sicht (CATCHLOVE, 2017, p. 17). Auf diese Weise wird eine konkrete faktische Unterstützung für die Parteien aufrechterhalten, zum Beispiel bei Problemen oder Fehlern im Netz. ${ }^{36 / 37}$ Da der Vertrag unabhängig von der digitalen Ausdrucksweise gesetzlich geregelt und geschützt ist, ist das physische Vertragsinstruments mit den Händen zu besitzen Garantie für die Parteien, juristische Instanzen in Anspruch nehmen zu können und somit die automatische Ausführung eines Vertrags abstellen, dessen Inhalt oder Ausführung durch Fehler infolge von Systemfehlern beeinträchtigt wurde (PERUGINI; CHECCO, 2016, p. 26-27).

Abschließend soll betont werden, dass die Thematik noch recht anfänglich ist. ${ }^{38}$ Mit der Zeit werden sich neue Auslegungen als unerlässlich erweisen, um die breite Anwendbarkeit von Smart Legal Contracts in der Domäne des deutschen Vertragsrechts zu ermöglichen. Auf diese Weise wird die Auferlegung von Sicherheitsmaßnahmen durch das Recht ${ }^{39}$ verstärkt, um so Engpässe und schädliche Rückschläge beim Einsatz dieser zu vermeiden und gleichzeitig das Spektrum ihrer Anwendungen zu schützen (JUELS; KOSBA; SHI, 2015, p. 15).

\subsection{Einsatzmöglichkeiten}

Bei Betrachtung des rechtlichen Konzepts Smart Legal Contracts lässt sich der Schluss ziehen, dass ihre Anwendungen extrem unterschiedlich sind. ${ }^{40}$ Der Versuch, die möglichen Anwendungen von Smart Legal Contracts in einer erschöpfenden Liste zu

35 Vgl. Heckelmann (2018, p. 510).

36 Es ist auch wichtig, hervorzuheben, dass Verträge, die mit dieser Technologie geschaffen werden, wie jede andere Softwareanwendung auch, nicht frei von technischen Fehlern und/oder Fehlfunktionen sind. Mit den Worten von Christian Kuss, ,Software ist nie fehlerfre“ (SASSENBERG; FABER, 2017, p. 314 et seq).

37 In Bezug auf diese Problematik mit Problemen oder Mängeln im Netzwerk, Fries (2018b, p. 90): „Die Vertragsparteien dürfen sich nicht blind auf eine funktionierende Software verlassen, sondern müssen ihre Vereinbarung jenseits der Software unmissverständlich niederlegen.“

38 Nicht nur die Smart Legal Contracts wie die selbige Blockchain-Technologie an sich. Vgl. Schrey; Thalhofer (2017, p. 1.436).

39 Nicht nur rechtliche, sondern auch politische Anstrengungen sind für den Aufbau eines Rechtsrahmens wesentlich, der aus Materie der Smart Legal Contracts und der Blockchain-Technologie entwickelt wurde. Vgl. Süme; Zimprich; Vogt (2018, p. 29).

$40 \mathrm{Zu}$ diesem Pluralismus von Anwendungen betreffend Smart Legal Contracts, siehe Schrey; Thalhofer (2017, p. 1.431). 
synthetisieren, wäre eine Unterschätzung ihrer eigenen Anwendbarkeit und zukünftigen Entwicklungsmöglichkeiten dieser Contracts. ${ }^{41}$ In diesem Sinne wird im Folgenden alles bisher zu dem Konzept und dem rechtlichen Szenario genannte veranschaulicht, was dieses neue Vertragsinstrument betrifft, welches, wie bereits untersucht, keinen eigenständigen Vertrag nach den Kriterien des BGB darstellt.

Der Prozess der Fertigstellung eines Vertrags durch Smart Legal Contracts hat im Allgemeinen folgende Vorgehensweise: ${ }^{42}$ (i) geeignete Vertragsklauseln werden in einen rechnerisch ausführbaren Code umgewandelt, (ii) die in der Blockchain registriert sind. (iii) Bei Auftreten einer bestimmten vorab festgelegten Bedingung oder bei Verletzung der vertraglichen Verpflichtungen, was beides mathematisch bestimmt ist, wird das Ereignis produziert, welches digital verbunden war. Dies bedeutet, dass, wenn das Einhalten innerhalb der verpflichtenden zwischenparteilichen Beziehung verifiziert wurde, automatisch die zuvor festgelegten Bedingungen des Geschäfts in Kraft treten, die seine Stabilität, Transparenz und Publizität garantieren.

Sinnbildliche Einsatzmöglichkeit und Pionier dieser Verträge sind die sogenannten Treuhandverträge (Escrow Contracts). Dies gilt als ein grundlegendes Beispiel für Smart Legal Contracts, das aufgrund seiner Einfachheit auf Bitcoins eigener Blockchain aufgebaut sein kann.

Der Escrow-Contract bietet, in dem Maße, in dem er sich als neutraler Vermittler etabliert, größeren Schutz für beide Vertragsparteien, indem er Nicht-Einhaltenoder möglicherweise schlechtes Befolgen der Vertragsbedingungen vermeidet. ${ }^{43}$

Ein Beispiel dazu kann das Szenario sein, bei dem der Käufer „K“ vom Verkäufer „V“ das Objekt „O' zum Preis von 2 ETH zu kaufen wünscht. Um dies in die Tat umzusetzen, erstellt „,V“ einen Smart Legal Contract und schreibt in seinen Inhalt die beabsichtigten Informationen (Verkaufsobjekt, akzeptierte Währung, Datum der Transaktion u.a.) gleichzeitig mit der Übertragung einer Summe von 4 ETH als Sicherheit. Der Situation entsprechend überträgt „K“ seinerseits zusätzlich zum Betrag, der für das Verkaufsobjekt „O“ (2 ETH) zu zahlen ist, auch den Betrag von 4 ETH als Vertragssicherheit - also „K“ “ transferiert insgesamt 6 ETH für den Vertrag. Wie untersucht, charakterisiert das Einhalten bei den Smart Legal Contracts das Akzeptieren des Vertrags

${ }_{41}$ Diese Entwicklung ist wesentlich, denn nach Fries gibt es im gegenwärtigen Kontext noch immer einen begrenzten Gebrauch von Smart Legal Contracts, die sich insbesondere auf die Bereiche von Geschäften mit virtuellen Währungen und Wertpapieren beschränken, vgl. Fries (2018b, p. 86). In diesem Sinne, siehe auch Rauscher; Cupic (2018, p. 7).

42 Siehe Breidenbach; Glatz (2018, p. 127).

43 Wie im vorherigen Kapitel untersucht, zeigt sich Bitcoins Blockchain als ein Werkzeug, das nicht in der Lage ist, die Konstruktion komplexer rechtlicher Verträge zu ermöglichen, sie unterscheidet sich daher von Ethereums Blockchain, vgl. Breidenbach; Glatz (2018, p. 72). 
und, sobald er akzeptiert ist, überträgt , $\mathrm{V}^{“}$, $\mathrm{O}^{\prime}$ auf „, $\mathrm{K}^{\prime}$. Nach Bestätigung des Erhaltens von „O“ erstattet der Vertrag den Parteien automatisch die hinterlegten Beträge (d.h. 4 ETH jeder Vertragspartei) und überweist darüber hinaus den Betrag, der dem Verkauf von „OO entspricht, an „V“ (nämlich 2 ETH).

Durch das Kombinieren von Smart Legal Contracts mit den Treuhandverträgen ${ }^{44}$ ergibt sich ein sicheres Umfeld für Verhandlung, die Übertragung von Vermögenswerten und die Zuteilung von Krypto-Münzen zwischen den beteiligten Parteien. Diese spezielle Anwendbarkeit betont die Tatsache, dass diese automatisierten Softwareprogramme nur in dem Maße automatisiert werden, in dem sich das Asset in Bezug auf Verwaltung und Ausführung auf der Blockchain-Plattform befindet. Tokenisieren von Assets (digitale Repräsentation von Objekt „O“) und die Verwendung von Kryptowährungen (in diesem Fall von ETH) sind für die Erfüllung dieser Verträge wesentlich, in dem Maße wie sie im dezentralen und kollektiven Netzwerk von Blockchain gelagert sind, und eben nicht in der physischen Welt (wie es sich z. B. bei einem nicht tokenisierten Objekt und einem Saldo in Euro auf einem Girokonto verhält).

Zusätzlich zu dieser Einsatzmöglichkeit von Smart Legal Contracts in Szenarien, in denen sie Escrow-Dienste oder Vermittler ersetzen, die für eine sichere Transaktion zwischen beiden Parteien notwendig sind, werden als eine zweite Anwendbarkeit Szenarien angeführt, die das Enforcement von Verbraucherrechten umfassen. Angesichts der Situation zum Beispiel, in der das Individuum „X“ ein Flugticket von der Fluggesellschaft ,Y“ kauft, sein Flug sich jedoch verspätet, könnte wieder daran gedacht werden, das Vertragsverhältnis zwischen den Parteien mittels Einsatz eines Smart Legal Contracts zu strukturieren, um das Enforcement von Vertragspositionen $\mathrm{zu}$ automatisieren und dadurch Verbraucherrecht $\mathrm{zu}$ garantieren. ${ }^{45} \mathrm{Zu}$ diesem Zweck würde „Y“ einen Vertrag bereitstellen, in dem über die Standardbedingungen hinaus beispielsweise eine Klausel über die Rückerstattung einer bestimmten Summe, welche pro Minute Flugverspätung auszuzahlen sei, enthalten wäre. Wie bei jedem Smart-LegalContract gibt es die Umwandlung von Informationen in geeigneter Computersprache und Registrierung in der Blockchain. „X“ seinerseits akzeptiert den Vertrag durch Tätigung des Ticketkaufs. Bei tatsächlich verspätetem Flug, ${ }^{46}$ wird der Smart Legal Contract

$44 \quad$ Siehe Süme; Zimprich; Vogt (2018, p. 27).

45 Ein solches Beispiel für die Anwendung Smart Legal Contracts im Rahmen der Beziehungen von Einzelpersonen mit Fluggesellschaften wurde von Privatdozent Dr. Martin Fries auf der 2. Jahreskonferenz des Oxford Business Law Blog am 08.03.2018 untersucht. In diesem Sinne, siehe Fries (2018a).

46 In diesem Beispiel, wie auch bereits vorher untersucht, muss die Teilnahme eines Orakels in den Vertrag aufgenommen werden, insofern, als die Interaktion mit der physischen Welt notwendig ist. In diesem Fall sind die Flight Tracker Beispiele von zweckmäßigen Orakeln, welche in ihrer Datenbank die Flugzeiten nach Plan mit den Flugzeiten, zu denen das Flugzeug tatsächlich gestartet ist abgleichen und alles überwachen. Es wird daher der gewählte Flight Tracker sein, der dem Smart Legal Contract mit der Information versorgt, 
unmittelbar ausgeführt, wobei „,X“ gemäß den Regeln des Codes entschädigt (oder Geld rückerstattet) wird.

Dieses Szenario von Anwendbarkeit Smart Legal Contracts in Verbindung mit Enforcement von Verbraucherrechten zeigt den Nutzen von vertraglicher Automatisierung auf. In solchen Verträgen kann es analog zu einem Vertrag, der in herkömmlicher Sprache abgefasst ist, das Festschreiben von Verpflichtungen und das Auferlegen von Konsequenzen geben, von Vorteil oder von Nachteil hinsichtlich vereinbarter Umstände. ${ }^{47}$ Solche vertraglichen Verpflichtungen ziehen ihrerseits keine zusätzlichen Kosten nach sich, was einen enormen Vorteil für den Verbraucher bedeutet. In der Tat wäre es das erste Mal, dass den Parteien einer bestimmten Vereinbarung die Gelegenheit gegeben wird, ihre vertraglichen Verpflichtungen durch einen sicheren und kostengünstigen externen Mechanismus zu automatisieren. In ihrer Konsequenz sind Vereinfachung, Automatisierung verschiedener Prozesse, Erhöhung von Standardisierung und darüber hinaus noch die Senkung der Transaktionskosten ${ }^{48}$ das Ergebnis dieser inhärenten Selbstausführung besagter Smart Legal Contracts.

Die potenziellen Anwendungsmöglichkeiten von Smart Legal Contracts umfassen eine große Auswahl von Szenarien, die sich nicht auf die hier untersuchten Bezüge beschränken. ${ }^{49}$ Zur Veranschaulichung sei die klare Anwendbarkeit dieser Verträge auch erwähnt für Situationen, in denen Versicherungspolicen, urheberrechtlich geschützte Inhalte, darüber hinaus finanzielle Operationen, die beispielsweise Wertpapiere und Derivate mit einbeziehen, und Arbeit mit dem Internet of Things (IoT) und dessen verschiedenen vernetzten Apparaten ${ }^{50}$ erforderlich sind. Alle diese Anwendungsmöglichkeiten, obwohl vom Markt ausgiebig- und zunehmend auch von der Lehre anerkannt, leiden jedoch immer noch unter der beträchtlichen Lücke von Forschung und spezifischem Wissen hinsichtlich ihrer Konformität und ihrer Wechselwirkung mit dem heutigen Vertragsrecht. ${ }^{51}$

\section{Fazit}

NachAbschluss derStudiebetreffend des Konzepts, derEinsatzmöglichkeiten im Recht und der rechtlichen Rahmenbedingungen der Smart Legal Contracts im

ob es eine Flugverspätung gegeben hat oder nicht.

47 Vgl. Bourque; Fung Ling Tsui (2014).

48 Vgl. Breidenbach; Glatz (2018, p. 111 et seq).

49 Siehe Heckmann; Schmid (2017, p. 15-16). Siehe auch Heckelmann (2018, p. 504-505).

$50 \quad$ Vgl. Hoffmann-Riem (2018, p. 26).

51 Vgl. Breidenbach; Glatz (2018, p. 117). 
Deutschen Recht, konnten im Hinblick auf dieses neue technologische Instrument auf Basis der Blockchain-Technologie einige Schlussfolgerungen gezogen werden.

Es wird daher bestätigt, dass Smart Legal Contracts die Facette von Smart Contracts sind, verwandt als Verträge im rechtlichen Sinne, d.h. implementiert werden, um eine vertragliche Rechtsbeziehung zu übersetzen. Wenn diese Situation verifiziert ist, bezieht sich die im Vertragsgebiet eingeführte Effizienz auf die Verifizierung, Ausführung und Durchsetzung der Bedingungen Kraft des Vertrags auf automatisierte Weise und garantiert gleichzeitig dessen Unveränderbarkeit, Unwiderruflichkeit und Transparenz. Attribute wie diese sind für eine höhere Vertragseffizienz- und Performance wesentlich, da insbesondere bei Vereinbarungen getroffen von unbekannten und anonymen Parteien sowohl das Bedürfnis nach Vertrauen in die Gegenpartei als auch die Interessen von zwischengeschalteten Dritten eliminiert werden.

Durch die Untersuchung der Besonderheiten der Anwendungen automatischer Softwareprogramme im juristischen Bereich wird darüber hinaus festgestellt, dass die Smart Legal Contracts keine neue vertragliche Form vertreten. Vielmehr geht es lediglich um mehr Anpassung der Art und Weise, in der die Parteien einen Vertrag schließen können, welcher als gültig vor der Deutschen Rechtsordnung gilt.

München, Oktober 2018.

\section{Literaturverzeichnis}

ANTONOPOULOS, Andreas. M. Mastering Bitcoin: unlocking digital cryptocurrencies. Sebastopol: O'Reilly Media, 2014.

ANTONOPOULOS, Andreas. M.; WOOD, Gavin. Mastering Ethereum: building smart contracts and dapps. Sebastopol: O’Reilly Media, 2018.

ATIYAH, Patrick. S. An introduction to the law of contract. 3. ed. Oxford: Clarendon Press, 1981.

AUNERT-MICUS, Shirley; GÜLLEMANN, Dirk; STRECKEL, Siegmar; TONNER, Norbert; WIESE, Ursula-Eva. Wirtschaftsprivatrecht: BGB Allgemeiner Teil, Schuldrecht, Sachenrecht, Handels- und Gesellschaftsrecht. München: Franz Vahlen, 2018.

BOURQUE, Samuel; TSUI, Sara Fung Ling. A lawyer's introduction to smart contracts. In: SCIENTIA NOBILITAT: Reviewed Legal Studies. Łask: [s. n.], 2014. p. 4-23.

BREIDENBACH, Stephan; GLATZ, Florian. Rechtshandbuch Legal Tech. München: C. H. Beck, 2018.

BUTERIN, Vitalik. A next-generation smart contract and decentralized application platform. Ethereum Whitepaper, [s. l.], 2014. Verfügbar unter: https://ethereum.org/whitepaper/. 
CATCHLOVE, Paul. Smart contracts: a new era of contract use. Social Science Research Network, Rochester, Dec. 2017. Verfügbar unter: https://papers.ssrn.com/sol3/papers.cfm?abstract_ $\mathrm{id}=3090226$.

DELMOLINO, Kevin; ARNETT, Mitchell; KOSBA, Ahmed; MILLER, Andrew; SHI, Elaine. Step by step towards creating a safe smart contract: lessons and insights from a cryptocurrency lab, 2015. Verfügbar unter: https://eprint.iacr.org/2015/460.pdf.

FARIA, José Eduardo. Juristas fora da curva: três perfis. Revista Direito GV, São Paulo, v. 12, n. 2, p. 272-310, 2016. Verfügbar unter: https://www.scielo.br/pdf/rdgv/v12n2/1808-2432-rdgv-12-2-0272. pdf.

FRIES, Martin. Man versus machine: using legal tech to optimize the rule of law. Social Science Research Network, Rochester, Sept. 2015. Verfügbar unter: https://papers.ssrn.com/sol3/papers. cfm?abstract_id=2842726.

FRIES, Martin. Smart consumer contracts: the end of civil procedure? Oxford, 2018a. (Law and Autonomous Systems Series). Verfügbar unter: https://www.law.ox.ac.uk/business-law-blog/ blog/2018/03/smart-consumer-contracts-end-civil-procedure.

FRIES, Martin. Smart Contracts: Brauchen schlaue Verträge noch Anwälte? Anwaltsblatt, n. 68, Heft 2, S. 86-90, 2018b. Verfügbar unter: https://www.researchgate.net/publication/328872972_ Smart_Contracts_Brauchen_schlaue_Vertrage_noch_Anwalte_Zusammenspiel_von_Smart_ Contracts_mit_dem_Beweismittelrecht_der_ZPO.

HECKELMANN, Martin. Zulässigkeit und Handhabung von Smart Contracts, NJW: Neue juristische Wochenschrift, München, S. 504-510, 2018.

HECKMANN, Dirk; SCHMID, Alexander. Blockchain und Smart Contracts. Recht und Technik im Überblick. Vereinigung der Bayerischen Wirtschaft, 2017. Verfügbar unter: https://www. vbw-bayern.de/Redaktion/Frei-zugaengliche-Medien/Abteilungen-GS/Wirtschaftspolitik/2019/ Downloads/190509-Blockchain-und-Smart-Contracts_neu.pdf.

HECKMANN, Jörn; KAULARTZ Markus. Selbsterfüllende Verträge: Smart Contracts: Quellcode als Vertragstext. $C^{\prime} t$ : Magazin für Computertechnik, Hanover, Heft 24, S. 138-140, 2016.

HOFFMANN-RIEM, Wolfgang. Die digitale Transformation als Herausforderung für die Legitimation rechtlicher Entscheidungen, In: UNGER, Sebastian; UNGERN-STERNBERG, Antje von. Demokratie und künstliche Intelligenz. Heidelberg: Mohr Siebeck, 2018. S. 129-160.

JUELS, Ari; KOSBA, Ahmed; SHI, Elaine. The ring of gyges: using smart contracts for crime. 2015. Verfügbar unter: https://eprint.iacr.org/2016/358.pdf.

KADAMANI, Rosine. Contratos x smart contracts, São Paulo, 2018. Verfügbar unter: https:// blockchainacademy.com.br/contratos-x-smart-contracts/. Abgerufen am: 18 Juni. 2018. 
KAULARTZ, Markus; HECKMANN, Jörn. Smart Contracts: Anwendungen der BlockchainTechnologie. Computer und Recht, Berlin, v. 32, n. 9, p. 618-624, 2016.

KOSBA, Ahmed; MILLER, Andrew; SHI, Elaine; WEN, Zikai; PAPAMANTHOU, Charalampos. Hawk: the blockchain model of cryptography and privacy-preserving smart contracts. [S. l.: s. n.], 2015. Verfügbar unter: https://eprint.iacr.org/2015/675.pdf.

LEE, Larissa. New kids on the blockchain: how bitcoin's technology could reinvent the stock market. Hastings Business Law Journal, San Francisco, CA, v. 12, n. 2, p. 81-132, Winter 2016.

LOSANO, Mario Giuseppe. Lições de informática jurídica. São Paulo: Resenha Tributária Ltda., 1974.

MAINELLI, Michael; MILNE, Alistair. The impact and potential of blockchain on the securities transaction lifecycle. Swift Institute, London, 9 May 2016. (Working Paper NO. 2015-007). Verfügbar unter: https://swiftinstitute.org/wp-content/uploads/2016/05/The-Impact-and-Potentialof-Blockchain-on-the-Securities-Transaction-Lifecycle_Mainelli-and-Milne-FINAL-1.pdf.

MCLEAN, Sue; DEANE-JOHNS, Simon. Demystifying Blockchain and distributed ledger technology: hype or hero? Computer Law Review International, Köln, v. 17, n. 4, p. 97-102, 2016.

MÖHLENKAMP, Magdalena; WESSEL, Tobias. Smart Contracts in der Versicherung: Chancen und rechtliche Herausforderungen, 2018. Verfügbar unter: https://www.wilhelm-rae.de/sites/ default/files/pdf/versicherungspraxis_-_smart_contracts_in_der_versicherung_-_mai_2018.pdf.

NAKAMOTO, Satoshi. Bitcoin: a peer-to-peer electronic cash system, 2008. Verfügbar unter: https://bitcoin.org/bitcoin.pdf.

PAECH, Philipp. Securities, intermediation and the blockchain: an inevitable choice between liquidity and legal certainty? Uniform Law Review, Oxford, v. 21, n. 4, p. 612-39, 2016.

PERUGINI, Maria Letizia; CHECCO, Paolo Dal. Introduzione agli smart contract. Social Science Research Network, Rochester, Feb. 2016. Verfügbar unter: https://papers.ssrn.com/sol3/papers. cfm?abstract_id=2729545.

PETERS, Gareth. W.; PANAYI, Efstathios. Understanding modern banking ledgers through blockchain technologies: future of transaction processing and smart contracts on the internet of money. In: TASCA, Paolo; ASTE, Tomaso; PELIZZON, Loriana; PERONY, Nicolas (ed.). Banking beyond banks and money: a guide to banking services in the twenty-first century. Berlin: Springer, 2015. p. 239-278.

QUIEL, Philipp. Blockchain-Technologie im Fokus von Art. 8 GRC und DS-GVO. DuD: Datenschutz und Datensicherheit, Berlin, v. 42, n. 9, S. 566-573, 2018.

RASKIN, Max. The law and legality of smart contracts. Georgetown Law Technology Review, Washington, D.C., v. 1, n. 2, p. 305-341, 2017. Verfügbar unter: https:/georgetownlawtechreview. org/wp-content/uploads/2017/05/Raskin-1-GEO.-L.-TECH.-REV.-305-.pdf. 
RAUSCHER, Andreas; CUPIC, Zoran. Blockchain bassierte Smart Contracts: Grundlagen, Prozessunterstützung und Bewertung, 2018. Verfügbar unter: https://www.syntax-solution.de/wpcontent/uploads/sites/7/2018/02/Blockhain-bassierte-Smart-Contract-Andreas-Rauscher-ZoranCupic.pdf.

ROPPO, Enzo. O contrato. Tradução de Ana Coimbra e M. Januário C. Gomes. Coimbra: Almedina, 2009.

ROUGHTON, Tim; BIDEWELL, Peter. Smart insurance contracts: A discussion paper by Pinsent Masons and Applied Blockchain on applications of blockchain, distributed ledger technology and smart contracts for the insurance sector, 2017. Verfügbar unter: https://www.the-digital-insurer. com/wp-content/uploads/2017/10/980-FinTech_Smart_Insurance_Contracts_Flyer.pdf.

SASSENBERG, Thomas; FABER, Tobias (ed.). Rechtshandbuch Industrie 4.0 und Internet of Thing: Praxisfragen und Perspektiven der digitalen Zukunft. München: Beck C. H, 2017.

SCHREY, Joachim; THALHOFER, Thomas. Rechtliche aspekte der blockchain. Neue Juristische Wochenschrift, München, Heft 20, S. 1.431-1.436, 2017.

SCHUMPETER, Joseph. A. Capitalism, socialism and democracy. New York: Harper \& Row, 1975.

SONG, Jimmt. The truth about smart contracts. [S. l.: s. n.], Jun. 2018. Verfügbar unter: https:// medium.com/@jimmysong/the-truth-about-smart-contracts-ae825271811f.

SÜME, Oliver; ZIMPRICH, Stephan; VOGT, Jan Niklas. Rechtliche Rahmenbedingungen der Blockchain. In: VDI TECHNOLOGIEZENTRUM GMBH. Blockchain: eine Technologie mit disruptivem Charakter, Technologiezentrum Gesellschaft für Informatik, 2018. S. 26-29. Verfügbar unter: https://www.vditz.de/fileadmin/media/bekanntmachungen/documents/vdi_publikation_ blockchain_RZ_web_neu.pdf.

SZABO, Nick. A formal language for analyzing contracts. [S. l.: $s . \quad n$.], 2002. Verfügbar unter: $\quad$ https://www.fon.hum.uva.nl/rob/Courses/InformationInSpeech/CDROM/Literature/ LOTwinterschool2006/szabo.best.vwh.net/contractlanguage.html.

SZABO, Nick. Contracts with bearers. [S. l.: s. n.], 1998. Verfügbar unter: https://www.fon.hum. uva.nl/rob/Courses/InformationInSpeech/CDROM/Literature/LOTwinterschool2006/szabo.best. vwh.net/bearer_contracts.html.

SZABO, Nick. Formalizing and securing relationships on public networks. First Monday, Copenhagen, v. 2, n. 9, Sept. 1997. Verfügbar unter: https://firstmonday.org/ojs/index.php/fm/ article/view/548.

SZABO, Nick. Secure property titles with owner authority. [S. l.: $s . \quad n$.$] , 1998. Verfügbar$ unter: https://www.fon.hum.uva.nl/rob/Courses/InformationInSpeech/CDROM/Literature/ LOTwinterschool2006/szabo.best.vwh.net/securetitle.html. 
SZABO, Nick. The idea of smart contracts. [S. l.: s. n.], 1997. Verfügbar unter: https://www.fon. hum.uva.nl/rob/Courses/InformationInSpeech/CDROM/Literature/LOTwinterschool2006/szabo. best.vwh.net/idea.html.

TAPSCOTT, Don; TAPSCOTT, Alex. Blockchain revolution: como a tecnologia por trás do Bitcoin está mudando o dinheiro, os negócios e o mundo. São Paulo: SENAI-SP, 2016.

WIEFLING, Stephan.; LO IACONO, Luigi; SANDBRINK, Frederik. Anwendung der Blockchain außerhalb von Geldwährungen. DuD: Datenschutz und Datensicherheit, Berlin, v. 41, n. 8, S. 482486, 2017.

WRIGHT, Aaron; DE FILIPPI, Primavera. Decentralized blockchain technology and the rise of lex cryptographia. Social Science Research Network, Rochester, 2015. Verfügbar unter: https://papers. ssrn.com/sol3/papers.cfm?abstract_id=2580664. 
